\title{
Nash equilibrium strategies and survival portfolio rules in evolutionary models of asset markets
}

DOI:

10.1007/s11579-019-00254-w

Document Version

Accepted author manuscript

Link to publication record in Manchester Research Explorer

\section{Citation for published version (APA):}

Belkov, S., Evstigneev, I. V., Hens, T., \& Xu, L. (2020). Nash equilibrium strategies and survival portfolio rules in evolutionary models of asset markets. Mathematics and Financial Economics. https://doi.org/10.1007/s11579-01900254-w

\section{Published in:}

Mathematics and Financial Economics

\section{Citing this paper}

Please note that where the full-text provided on Manchester Research Explorer is the Author Accepted Manuscript or Proof version this may differ from the final Published version. If citing, it is advised that you check and use the publisher's definitive version.

\section{General rights}

Copyright and moral rights for the publications made accessible in the Research Explorer are retained by the authors and/or other copyright owners and it is a condition of accessing publications that users recognise and abide by the legal requirements associated with these rights.

\section{Takedown policy}

If you believe that this document breaches copyright please refer to the University of Manchester's Takedown Procedures [http://man.ac.uk/04Y6Bo] or contact uml.scholarlycommunications@manchester.ac.uk providing relevant details, so we can investigate your claim.

\section{OPEN ACCESS}




\title{
Nash Equilibrium Strategies and Survival Portfolio Rules in Evolutionary Models of Asset Markets
}

\author{
Sergei Belkov • Igor V. Evstigneev • \\ Thorsten Hens • Le Xu
}

Received: date / Accepted: date

\begin{abstract}
We consider a stochastic model of a financial market with oneperiod assets and endogenous asset prices. The model was initially developed and analyzed in the context of Evolutionary Finance with the main focus on questions of "survival and extinction" of investment strategies (portfolio rules). In this paper we view the model from a different, game-theoretic, perspective and analyze Nash equilibrium properties of survival portfolio rules.
\end{abstract}

Keywords Stochastic games · Evolutionary finance - Capital growth theory · Random dynamical systems

JEL classification $\mathrm{C} 73 \cdot \mathrm{D} 52 \cdot \mathrm{G} 11$

S. Belkov

Economics Department, University of Manchester

Oxford Road, Manchester M13 9PL, UK

E-mail: serg.belkov@gmail.com

I. V. Evstigneev

Economics Department, University of Manchester

Oxford Road, Manchester M13 9PL, UK

E-mail: igor.evstigneev@manchester.ac.uk

T. Hens (Corresponding author)

Department of Banking and Finance, University of Zurich and Swiss Finance Institute Plattenstr. 32, 8032 Zurich, Switzerland

E-mail: Thorsten.Hens@bf.uzh.ch

T. Hens

Department of Finance and Management Science, NHH - Norwegian School of Economics 5045 Bergen, Norway

L. Xu

Department of Strategy and Policy, NUS Business School, National University of Singapore

15 Kent Ridge Drive, Singapore 119245, Singapore

E-mail: bizxl@nus.edu.sg 


\section{Introduction}

This paper examines a stochastic model of a financial market with shortlived (one-period) assets and endogenous market clearing asset prices. The model is a version of that developed in Evstigneev et al. [9] and Amir et al. [2] in the framework of Evolutionary Finance. Surveys on Evolutionary Finance and related questions are given in $[11,13]$; for an elementary textbook treatment see [12], Ch. 20. The main focus in the field is on questions of "survival and extinction" of investment strategies. The fundamental results show that one can identify an asymptotically unique portfolio rule $\Lambda^{*}$ which guarantees unconditional survival of an investor almost surely over an infinite time horizon, irrespective of the strategies used by all the other investors. Here, "survival" means a possibility of keeping strictly positive bounded away from zero fraction of total market wealth over an infinite time interval.

In this paper we analyze the Evolutionary Finance model from a different, game-theoretic, perspective. We consider a game related to this model in which the payoffs of the players/investors are defined in terms of the growth rates of their relative wealth. We show that in the game under consideration the strategy $\Lambda^{*}$ forms with probability one a unique symmetric Nash equilibrium.

Game-theoretic models of asset markets dealing with relative wealth of investors were considered by Bell and Cover $[4,5]$. In those models, the objectives of the players were described in terms of the expectations of random payoffs. The notion of Nash equilibrium defined in terms of these expectations was used as a game solution concept. We consider a different (stronger) solution concept: almost sure Nash equilibrium. According to our definition of an equilibrium strategy, any unilateral deviation from it leads to a decrease in the random payoff with probability one, and not only to a decrease in the expected payoff.

The focus on optimality almost surely, understood in one sense or another, is characteristic for capital growth theory (Kelly [16], Latané [18], Breiman [6], Algoet and Cover [1], Hakansson and Ziemba [14], MacLean et al. [19], Kuhn and Luenberger [17], Ziemba and Vickson [21], MacLean and Ziemba [20], and others $)^{1}$, dealing in its classical version with exogenous asset prices. Results related to Evolutionary Finance models may be regarded as analogues, and in certain cases as generalizations, of those pertaining to classical models of capital growth. A fundamental distinction between the two modelling frameworks lies in the fact that in the former the dynamics of wealth of an investor might depend (via the endogenous price formation mechanism) not only on his/her strategy, but also on the strategies used by the other investors. Therefore in

\footnotetext{
1 Although Claude Shannon-the famous founder of the mathematical theory of information - did not publish on investment-related issues, his ideas, expressed in his lectures on investment problems (MIT 1956), should be regarded as the initial source of the strand of literature we cite here. For the history of these ideas and the related discussion see Cover [7]. An excellent exposition of capital growth theory is given in Ch. 16 "Information Theory and Portfolio Theory" in the book by Cover and Thomas [8]. For an elementary introduction to the subject see Evstigneev et al. [12], Ch. 17.
} 
the present context a game-theoretic model, rather than a single-agent optimization framework, is a suitable setting for the analysis of questions related to capital growth.

The paper is organized as follows. Section 2 describes the model, Section 3 states the main results and Section 4 gives the proof of the main theorem. The Appendix contains the proof of a technical lemma.

\section{The model}

There are $N \geq 2$ investors (traders) acting in a market where $K \geq 2$ risky assets (securities) $k=1,2, \ldots$, are traded. The total amount of security $k$ in the market is $V_{k}>0$. A portfolio of investor $i$ at date $t=0,1, \ldots$ is characterized by a vector $x_{t}^{i}:=\left(x_{t, 1}^{i}, \ldots, x_{t, K}^{i}\right) \in \mathbb{R}_{+}^{K}$ where $x_{t, k}^{i}$ indicates the amount ("physical units") of asset $k$ in the portfolio $x_{t}^{i}$. We denote by $p_{t} \in \mathbb{R}_{+}^{K}$ the vector of market prices of the securities. For each $k=1, \ldots, K$, the coordinate $p_{t, k}$ of $p_{t}:=\left(p_{t, 1}, \ldots, p_{t, K}\right)$ stands for the price of one unit of asset $k$ at date $t$. The scalar product $\left\langle p_{t}, x_{t}^{i}\right\rangle:=\sum_{k=1}^{K} p_{t, k} x_{t, k}^{i}$ expresses the market value of investor $i$ 's portfolio at date $t$.

Random factors influencing the market are described in terms of an exogenous stochastic process $s_{1}, s_{2}, \ldots$ with values in a measurable space $S$. The random element $s_{t}$ in the space $S$ represents the "state of the world" at date $t$. Throughout this paper, we will assume that the random elements $s_{t}, t=1,2, \ldots$, are independent and identically distributed (i.i.d.).

Assets live for one period: they are traded at the beginning of the period and yield payoffs at the end of it; then the cycle repeats. The payoff $A_{t, k}=$ $A_{k}\left(s_{t}\right) \geq 0$ of asset $k=1,2, \ldots, K$ at date $t=1,2, \ldots$ depends on the state of the world $s_{t}$ at that date. The functions $A_{k}(s), s \in S$, are measurable and satisfy

$$
\sum_{k=1}^{K} A_{k}(s)>0 \text { for all } s .
$$

The last inequality means that in each random situation at least one asset gives a strictly positive payoff.

At date $t=0$ investors have initial endowments $w_{0}^{i}>0(i=1,2, \ldots, N)$. These initial endowments form the traders' budgets at date 0 . Trader $i$ 's budget at date $t \geq 1$ is

$$
w_{t}^{i}=\left\langle A_{t}, x_{t-1}^{i}\right\rangle, \text { where } A_{t}:=\left(A_{t, 1}, \ldots, A_{t, K}\right) .
$$

It is formed by the payoffs of the assets contained in yesterday's portfolio $x_{t-1}^{i}$ of investor $i$. This budget is re-invested in the assets available at date $t$, that will yield payoffs $A_{t+1, k}=A_{k}\left(s_{t+1}\right), k=1, \ldots, K$, at the next date $t+1$.

For every $t \geq 0$, each trader $i=1,2, \ldots, N$ selects a vector of investment proportions $\lambda_{t}^{i}:=\left(\lambda_{t, 1}^{i}, \ldots, \lambda_{t, K}^{i}\right)$ according to which she plans to distribute the available budget between assets. Vectors $\lambda_{t}^{i}$ belong to the unit simplex

$$
\Delta^{K}:=\left\{\left(a_{1}, \ldots, a_{K}\right) \in \mathbb{R}_{+}^{K}: a_{1}+\ldots+a_{K}=1\right\} .
$$


The investment proportions at each date $t \geq 0$ are selected by the $N$ traders simultaneously and independently (so that we deal here with a simultaneousmove $N$-person dynamic game). They represent in the game under consideration the players' actions or decisions. For $t \geq 1$, these decisions might depend, generally, on the history

$$
s^{t}:=\left(s_{1}, \ldots, s_{t}\right)
$$

of the process of states of the world. A vector $\lambda_{0}^{i} \in \Delta^{K}$ and a sequence of measurable functions $\lambda_{t}^{i}\left(s^{t}\right), t=1,2, \ldots$, with values in $\Delta^{K}$ form a portfolio rule, or an investment (trading) strategy $\Lambda^{i}$ of trader $i$, according to which trader $i$ selects investment proportions at each date $t \geq 0$.

Suppose each investor $i$ at date 0 has selected investment proportions $\lambda_{0}^{i}=$ $\left(\lambda_{0,1}^{i}, \ldots, \lambda_{0, K}^{i}\right) \in \Delta^{K}$. Then the amount invested in asset $k$ by trader $i$ will be $\lambda_{0, k}^{i} w_{0}^{i}$, and the total amount invested in asset $k$ will be equal to $\sum_{i=1}^{N} \lambda_{0, k}^{i} w_{0}^{i}$. The equilibrium price $p_{0, k}$ of each asset $k$ can be determined from the equations

$$
p_{0, k} V_{k}=\sum_{i=1}^{N} \lambda_{0, k}^{i} w_{0}^{i}, k=1,2, \ldots, K .
$$

On the left-hand side of (2), we have the total value, expressed in terms of the price $p_{0, k}$, of the assets of the $k$ th type purchased by the market participants at date 0 (recall that the amount of each asset $k$ at date 0 is $V_{0, k}$ ). On the right-hand side, we have the total sum of money invested in asset $k$ by all the investors.

The investors' portfolios $x_{0}^{i}=\left(x_{0,1}^{i}, \ldots, x_{0, K}^{i}\right), i=1,2, \ldots, N$, at date 0 can be determined from the equations

$$
x_{0, k}^{i}=\frac{\lambda_{0, k}^{i} w_{0}^{i}}{p_{0, k}}, k=1,2, \ldots, K, i=1, \ldots, N,
$$

meaning that the current market value $p_{0, k} x_{t, k}^{i}$ of the $k$ th position of the portfolio $x_{t}^{i}$ is equal to the fraction $\lambda_{0, k}^{i}$ of the trader $i$ 's investment budget $w_{0}^{i}$.

Suppose now that all the investors have chosen their investment proportion vectors $\lambda_{t}^{i}=\left(\lambda_{t, 1}^{i}, \ldots, \lambda_{t, K}^{i}\right)$ at date $t \geq 1$. Then the balance between aggregate asset supply and demand implies the formula determining the equilibrium prices

$$
p_{t, k} V_{k}=\sum_{i=1}^{N} \lambda_{t, k}^{i}\left\langle A_{t}, x_{t-1}^{i}\right\rangle, k=1, \ldots, K,
$$

which, in turn, yields the expression for the investors' portfolios $x_{t}^{i}=\left(x_{t, 1}^{i}, \ldots\right.$, $\left.x_{t, K}^{i}\right)$ :

$$
x_{t, k}^{i}=\frac{\lambda_{t, k}^{i}\left\langle A_{t}, x_{t-1}^{i}\right\rangle}{p_{t, k}}, k=1, \ldots, K, i=1, \ldots, N .
$$

Here, in contrast with the case $t=0$, the traders' budgets

$$
w_{t}^{i}=\left\langle A_{t}, x_{t-1}^{i}\right\rangle
$$


at date $t \geq 1$ are not given exogenously as initial endowments, rather they are formed by the payoffs of the previous date's portfolios $x_{t-1}^{i}$.

Given a strategy profile $\left(\Lambda^{1}, \ldots, \Lambda^{N}\right), \Lambda^{i}=\left\{\lambda_{t}^{i}\left(s^{t}\right)\right\}_{t=0}^{\infty}$, of the investors, we can generate a path of the market game by defining $p_{t}$ and $x_{t}^{i}$ recursively according to equations (2)-(5). The random dynamical system described determines the state of the market at each moment of time $t \geq 1$ as a measurable vector function of $s^{t}$ :

$$
\left(p_{t}\left(s^{t}\right) ; x_{t}^{1}\left(s^{t}\right), \ldots, x_{t}^{N}\left(s^{t}\right) ; \lambda_{t}^{1}\left(s^{t}\right), \ldots, \lambda_{t}^{N}\left(s^{t}\right)\right),
$$

where $p_{t}\left(s^{t}\right), x_{t}^{i}\left(s^{t}\right)$ and $\lambda_{t}^{i}\left(s^{t}\right)$ are the vectors of equilibrium prices, investors' portfolios and their investment proportions, respectively. (For $t=0$, the vectors $p_{t}, x_{t}^{i}$ and $\lambda_{t}^{i}$ are constant.)

A comment regarding the above description of asset market dynamics is in order. Portfolio positions $x_{t, k}^{i}$ are well-defined by formulas (3) and (5) only if the prices $p_{t, k}$ are strictly positive for $t \geq 0$, or equivalently, if the aggregate demand $\sum_{i=1}^{N} \lambda_{t, k}^{i} w_{t}^{i}$ for each asset $k$ is strictly positive:

$$
\sum_{i=1}^{N} \lambda_{0, k}^{i} w_{0}^{i}>0 \text { and } \sum_{i=1}^{N} \lambda_{t, k}^{i}\left\langle A_{t}, x_{t-1}^{i}\right\rangle>0
$$

Those strategy profiles for which the recursive procedure defined above generates non-zero aggregate demand for each asset will be called admissible. In what follows, we will deal only with strategy profiles satisfying this assumption. This assumption guarantees that the random dynamical system under consideration is well-defined and $p_{t, k}>0$ for all $t$ and $k$. By summing up equations (3) or (5) over $i=1, \ldots, N$, we find that

$$
\sum_{i=1}^{N} x_{0, k}^{i}=\frac{\sum_{i=1}^{N} \lambda_{0, k}^{i} w_{0}^{i}}{p_{0, k}}=\frac{p_{0, k} V_{k}}{p_{0, k}}=V_{k}
$$

and

$$
\sum_{i=1}^{N} x_{t, k}^{i}=\frac{\sum_{i=1}^{N} \lambda_{t, k}^{i}\left\langle A_{t}, x_{t-1}^{i}\right\rangle}{p_{t, k}}=\frac{p_{t, k} V_{k}}{p_{t, k}}=V_{k},
$$

i.e., the market clears for every asset $k$ and each date $t \geq 0$. It follows from this formula that the total market wealth

$$
W_{t}:=w_{t}^{1}+\ldots+w_{t}^{N}
$$

can be expressed as follows:

$$
W_{t}=\sum_{i=1}^{N}\left\langle A_{t}, x_{t-1}^{i}\right\rangle=\sum_{i=1}^{N} \sum_{i=1}^{K} A_{t, k} x_{t-1, k}^{i}=\sum_{i=1}^{K} A_{t, k} \sum_{i=1}^{N} x_{t-1, k}^{i}=\sum_{i=1}^{K} A_{t, k} V_{k}
$$

which implies by virtue of (1) that $W_{t}>0$.

We provide a sufficient condition for a strategy profile to be admissible, that will hold for all the strategy profiles we consider in the present paper (it 
will follow from (13) and (14)). Assume that one of the traders, e.g., trader 1 , employs a fully diversified portfolio rule, prescribing to invest into all the assets in strictly positive proportions $\lambda_{t, k}^{1}$. Then any strategy profile containing this portfolio rule is admissible. Indeed, for $t=0$, we get from (2) that $p_{0, k} \geq$ $V_{k}^{-1} \lambda_{0, k}^{1} w_{0}^{1}>0$ and from (3) that $x_{0}^{1}=\left(x_{0,1}^{1}, \ldots, x_{0, K}^{1}\right)>0$ (coordinatewise). Assuming that $x_{t-1}^{1}>0$ and arguing by induction, we obtain

$$
w_{t}^{1}=\left\langle A_{t}, x_{t-1}^{1}\right\rangle>0
$$

in view of (1), which in turn yields $p_{t}>0$ and $x_{t}^{1}>0$ by virtue of (4) and (5), as long as $\lambda_{t, k}^{1}>0$.

Key results related to the present model obtained in Amir et al. [2] in a substantially more general setting are concerned with the description of survival investment strategies. A strategy $\Lambda^{i}$ of trader $i$ is called a survival strategy or a survival portfolio rule if for any strategies $\Lambda^{j}$ of investors $j \neq i$ the market share

$$
r_{t}^{i}:=\frac{w_{t}^{i}}{w_{t}^{1}+\ldots+w_{t}^{N}}
$$

of trader $i$ is strictly positive and bounded away from zero almost surely:

$$
\inf _{t} r_{t}^{i}>0 \text { (a.s.). }
$$

Define the relative payoffs by

$$
R_{k}(s):=\frac{A_{k}(s) V_{k}}{\sum_{m=1}^{K} A_{m}(s) V_{m}}
$$

and put

$$
\lambda_{k}^{*}=E R_{k}\left(s_{t}\right), k=1, . ., K .
$$

Clearly $E R_{k}\left(s_{t}\right)$ does not depend on $t$ because $s_{t}$ are independent and identically distributed. Consider the strategy or which the vector of investment proportions $\lambda_{t}^{*}=\lambda^{*}=\left(\lambda_{1}^{*}, \ldots, \lambda_{K}^{*}\right)$ is constant (independent of $t$ and $\left.s^{t}\right)$ and the numbers $\lambda_{k}^{*}, k=1, \ldots, K$, are given by formula (13). The portfolio rule specified by (13) prescribes to distribute wealth across assets in accordance with the proportions of the expected relative payoffs, which do not depend on $t$ because the random elements $s_{t}$ are i.i.d..Strategies of this kind are called fixed-mix, or constant proportions, portfolio rules: they prescribe to select investment proportions at time 0 and keep them fixed over the whole infinite time horizon.

Assume that

$$
E R_{k}\left(s_{t}\right)>0
$$

for each $k=1,2, \ldots, K$. Central results related to the present Evolutionary Finance model are as follows.

Theorem 1. The portfolio rule $\Lambda^{*}$ is a survival strategy.

Note that although $\Lambda^{*}$ is a fixed-mix strategy, it guarantees unconditional survival in competition with all, not necessarily fixed-mix strategies. 
The following theorem shows that the survival strategy $\Lambda^{*}=\left(\lambda_{t}^{*}\right)$ is essentially unique: any other survival portfolio rule is in a sense asymptotically similar to $\Lambda^{*}$.

Theorem 2. If $\Lambda=\left(\lambda_{t}\right)$ is a survival strategy, then

$$
\sum_{t=0}^{\infty}\left\|\lambda_{t}^{*}-\lambda_{t}\right\|^{2}<\infty \text { (a.s.). }
$$

For proofs these results see Theorems 1 and 2 in [2].

\section{The main results}

In this paper, we view the present model from a more traditional gametheoretic standpoint and analyze Nash equilibrium properties of the survival strategy $\Lambda^{*}$. From now on we will focus exclusively on fixed-mix strategies, and a "strategy" ("portfolio rule") will always mean a "fixed-mix strategy" ("fixed-mix portfolio rule"). Thus by a strategy we will mean just a vector $\lambda$ of investment proportions.

Let $\left(\lambda^{1}, \ldots, \lambda^{N}\right)$ be an admissible strategy profile of the investors. Consider the path (7) of the random dynamical system generated by this strategy profile and the given initial endowments. As above, let $w_{t}^{i}$ denote the investor $i$ 's wealth available at date $t \geq 0$. If $t=0$, then $w_{0}^{i}$ is a constant number, the initial endowment of investor $i$. If $t \geq 1$, then $w_{t}^{i}=w_{t}^{i}\left(s^{t}\right)$ is a measurable function of $s^{t}$ given by formula (6). As we have noted above $w_{t}^{1}+\ldots+w_{t}^{N}>0$.

Given a strategy profile $\left(\lambda^{1}, \ldots, \lambda^{N}\right)$, the performance of a strategy $\lambda^{i}$ used by investor $i$ will be characterized by the following random variable

$$
\xi^{i}:=\limsup _{t \rightarrow \infty} \frac{1}{t} \ln \frac{w_{t}^{i}}{\sum_{j \neq i} w_{t}^{j}},
$$

generally, taking values in $[-\infty,+\infty]$. The expression $w_{t}^{i} / \sum_{j \neq i} w_{t}^{j}$ is the relative wealth of player $i$ and the group $\{j: j \neq i\}$ of $i$ 's rivals. The random variable $\xi^{i}=\xi^{i}\left(s^{\infty} ; \lambda^{1}, \ldots, \lambda^{N}\right)$ depends on the strategy profile $\left(\lambda^{1}, \ldots, \lambda^{N}\right)$ and on the whole history $s^{\infty}:=\left(s_{1}, s_{2}, \ldots\right)$ of states of the world from time 1 to $\infty$. In the game under consideration, $\xi^{i}$ plays the role of the (random) payoff function of player $i$.

We shall say that a strategy $\bar{\lambda}$ forms a symmetric Nash equilibrium almost surely (a.s.) if

$$
\xi^{i}\left(s^{\infty} ; \bar{\lambda}, \ldots, \bar{\lambda}\right) \geq \xi^{i}\left(s^{\infty} ; \bar{\lambda}, \ldots, \lambda, \ldots, \bar{\lambda}\right)(\text { a.s. })
$$

for every $i$, each strategy $\lambda$ of investor $i$ and each set of initial endowments $w_{0}^{1}>0, \ldots, w_{0}^{N}>0$. The Nash equilibrium is called strict if the inequality in (17) is strict for any $\bar{\lambda}$.

Recall that we consider only those strategy profiles which are admissible. Clearly, if all the players use the same strategy $\bar{\lambda}$, then the strategy profile 
$(\bar{\lambda}, \ldots, \bar{\lambda})$ is admissible if and only if the vector $\bar{\lambda}$ is strictly positive (see $(8))$. Let the following assumption hold.

The functions $A_{1}(s), \ldots, A_{K}(s)$ are linearly independent with respect to the probability distribution of $s_{t}$, i.e. the equality $\sum \gamma_{k} A_{k}\left(s_{t}\right)=0$ holding a.s. for some constants $\gamma_{k}$ implies that $\gamma_{1}=\ldots=\gamma_{K}=0$.

Theorem 3. The portfolio rule $\lambda^{*}$ forms a unique symmetric Nash equilibrium almost surely. If an investor $i$ uses any strategy $\lambda$ distinct from $\lambda^{*}$, then

$$
\xi^{i}\left(s^{\infty} ; \lambda^{*}, \ldots, \lambda, \ldots, \lambda^{*}\right)<\xi^{i}\left(s^{\infty} ; \lambda^{*}, \ldots, \lambda^{*}\right)=0 \text { (a.s.) }
$$

and so the Nash equilibrium formed by the strategy $\lambda^{*}$ is strict.

The uniqueness of $\lambda^{*}$ is understood in the following sense: if $\lambda$ is a portfolio rule forming a symmetric Nash equilibrium a.s., then $\lambda=\lambda^{*}$.

Theorem 3 implies the following property of the portfolio rule $\lambda^{*}$ (cf. Evstigneev et al. [9]). If all the investors except one, say investor $i$, use the strategy $\lambda^{*}$ and $i$ uses any other strategy $\lambda$ distinct from $\lambda^{*}$, then the relative wealth $w_{t}^{i} / \sum_{j \neq i} w_{t}^{j}$ of $i$ tends to zero at the exponential rate $\xi^{i}<0$ (a.s.). In other words, the group of investors using the portfolio rule $\lambda^{*}$ drives all the others out of the market, which is interpreted in Evolutionary Finance as the property of global (holding for all initial states) evolutionary stability of $\lambda^{*}$.

For a different Evolutionary Finance model, dealing with long-lived dividendpaying assets (see Evstigneev et al. [10]), the counterpart of Theorem 3 was obtained in Bahsoun et al. [3]).

We conclude this section with a comment on the definition of the Lyapunov exponent (16) in terms of the variables $w_{t}^{i} / \sum_{j \neq i} w_{t}^{j}$ expressing the relative wealth of player $i$ and the group $\{j: j \neq i\}$ of $i$ 's rivals. In the Evolutionary Finance literature, relative wealth is typically defined as $r_{t}^{i}=w_{t}^{i} / \sum_{j=1}^{N} w_{t}^{j}$ (the market share of investor $i$ ). In many cases, results can be equivalently formulated both in terms of relative wealth - as it is defined in this paper and market shares. But this is not the case in the context of the present work. The consideration of the Lyapunov exponent

$$
\eta^{i}:=\lim \sup _{t \rightarrow \infty} \frac{1}{t} \ln \frac{w_{t}^{i}}{\sum_{j=1}^{N} w_{t}^{j}}
$$

leads to a trivial notion of a Nash equilibrium. If the payoff functions of the players $i=1, . ., N$ in the market game are defined by (19), then any completely mixed strategy $\lambda$ forms a symmetric Nash equilibrium. Indeed, $\eta^{i}(\lambda, \ldots, \lambda)=0$ because if all the investors use the same strategy, their market shares remain constant. On the other hand, $\eta^{i}$ is always non-positive, and so $\eta^{i}\left(\lambda^{1}, \ldots, \lambda^{K}\right) \leq$ $0=\eta^{i}(\lambda, \ldots, \lambda)$, which implies that the strategy profile $(\lambda, \ldots, \lambda)$ is a Nash equilibrium. 


\section{Proof of the main result}

The proof of Theorem 3 is based on an auxiliary result, Lemma 1 below. For any $\lambda=\left(\lambda_{1}, \ldots, \lambda_{K}\right) \in \Delta^{K}$ and $\kappa \in(0,1]$ put

$$
F(\lambda, \kappa, s):=\frac{\sum_{k=1}^{K} R_{k}(s) \frac{\lambda_{k}^{*}}{\lambda_{k}^{*} \kappa+\lambda_{k}(1-\kappa)}}{\sum_{k=1}^{K} R_{k}(s) \frac{\lambda_{k}}{\lambda_{k}^{*} \kappa+\lambda_{k}(1-\kappa)}},
$$

where the numbers $\lambda_{k}^{*}>0$ are defined by (13). Observe that the nominator $A$ of the fraction $F(\lambda, \kappa, s)=: A / B$ is not less than

$$
c:=\min \left\{\lambda_{1}^{*}, \ldots, \lambda_{K}^{*}\right\}
$$

while the denominator $B$ is not greater than $c^{-1}$. Therefore

$$
c^{2} \leq F(\lambda, \kappa, s) \leq+\infty .
$$

Lemma 1. For any $\lambda \in \Delta^{K}$ distinct from $\lambda^{*}$ there exist numbers $H>0$ and $\delta>0$ such that

$$
E \min \{H, \ln F(\lambda, \kappa, s)\} \geq \delta
$$

for all $\kappa \in(0,1]$.

The proof of this lemma is routine but rather lengthy, therefore it is relegated to the Appendix.

A comment about the function $F(\lambda, \kappa, s)$ is in order. An expression involving $F$ appears in the random dynamical system (23) analyzed in the course of the proof of Theorem 3. A key step in the proof lies in the application of the law of large numbers to the sequence of martingale differences $G_{t}^{H}-E_{t-1} G_{t}^{H}$, where $G_{t}^{H}$ is defined in terms of the truncation of $F$ by some constant $H$ (see (26)). As is well known, the finiteness of expectations is not enough for the validity of this version of the law of large numbers, but the boundedness of the random variables is fully sufficient - hence the truncation of $F$ by $H$.

Proof of Theorem 3. In order to prove Theorem 3 it is sufficient to consider the case of two investors $(N=2)$ because the group of $N-1$ investors who all use $\lambda^{*}$ is equivalent in terms of wealth dynamics to one, using the strategy $\lambda^{*}$ and possessing the total wealth of the group (see [2], p. 133). Suppose investors 1 and 2 use the strategies $\lambda^{*}$ and $\lambda \neq \lambda^{*}$, respectively. Put

$$
z_{t}:=w_{t}^{1} / w_{t}^{2}=r_{t}^{1} / r_{t}^{2}
$$

Since $\lambda^{*}>0$, the variable $z_{t}$ takes on its values in $(0,+\infty]$. The sequence $\left(z_{t}\right)$ satisfies

$$
z_{t+1}=z_{t} \frac{\sum_{k=1}^{K} R_{t+1, k} \frac{\lambda_{k}^{*}}{\lambda_{k}^{*} r_{t}^{1}+\lambda_{k}\left(1-r_{t}^{1}\right)}}{\sum_{k=1}^{K} R_{t+1, k} \frac{\lambda_{k}}{\lambda_{k}^{*} r_{t}^{1}+\lambda_{k}\left(1-r_{t}^{1}\right)}}
$$


([2], p. 133). To verify (18), it suffices to show that

$$
\liminf _{t \rightarrow \infty} t^{-1} \ln z_{t}>0 \text { (a.s.). }
$$

Indeed, if we know that (24) holds, then

$$
\begin{gathered}
\xi^{2}\left(\lambda^{*}, \lambda\right)=\limsup _{t \rightarrow \infty} t^{-1} \ln \frac{w_{t}^{2}}{w_{t}^{1}}=\limsup _{t \rightarrow \infty} t^{-1} \ln \frac{r_{t}^{2}}{r_{t}^{1}}=\limsup _{t \rightarrow \infty}\left(-t^{-1} \ln z_{t}\right)= \\
=-\liminf _{t \rightarrow \infty}\left(t^{-1} \ln z_{t}\right)<0=\xi^{2}\left(\lambda^{*}, \lambda^{*}\right) \text { (a.s.). }
\end{gathered}
$$

Let us verify (24). Put $G_{t}=\ln z_{t}-\ln z_{t-1}$. Then

$$
\sum_{t=1}^{T} G_{t}=\sum_{t=1}^{T}\left(\ln z_{t}-\ln z_{t-1}\right)=\ln z_{T}-\ln z_{0} .
$$

Hence it suffices to prove that

$$
\liminf _{T \rightarrow \infty} T^{-1} \sum_{t=1}^{T} G_{t}>0 \text { (a.s.). }
$$

For any constant $H$ define $G_{t}^{H}:=\min \left(G_{t}, H\right)$. Since $G_{t}^{H} \leq G_{t}$ it is sufficient to show that

$$
\liminf _{T \rightarrow \infty} T^{-1} \sum_{t=1}^{T} G_{t}^{H}>0 \text { (a.s.) }
$$

for some $H$. We have

$$
G_{t+1}=\ln z_{t+1}-\ln z_{t}=\ln \frac{\sum_{k=1}^{K} R_{t+1, k} \frac{\lambda_{k}^{*}}{\lambda_{k}^{*} r_{t}^{1}+\lambda_{k}\left(1-r_{t}^{1}\right)}}{\sum_{k=1}^{K} R_{t+1, k} \frac{\lambda_{k}}{\lambda_{k}^{*} r_{t}^{1}+\lambda_{k}\left(1-r_{t}^{1}\right)}}=\ln F\left(\lambda, r_{t}^{1} ; s_{t+1}\right)
$$

and

$$
G_{t+1}^{H}\left(s^{t+1}\right)=\min \left\{H, \ln F\left(\lambda, r_{t}^{1}\left(s^{t}\right) ; s_{t+1}\right)\right\} .
$$

By virtue of Lemma 1 , there exist $H>0$ and $\delta>0$, such that $E_{t} G_{t+1}^{H} \geq \delta$, where $E_{t}(\cdot)=E\left(\cdot \mid s^{t}\right)$ is the conditional expectation given $s^{t}$. When computing $E_{t} G_{t+1}^{H}$ we fix $s^{t}$ and take the unconditional expectation of $G_{t+1}^{H}$ with respect to $s_{t+1}$, which is justified because $s^{t}$ and $s_{t+1}$ are independent.

Finally, we have

$$
\frac{1}{T} \sum_{t=1}^{T} G_{t}^{H}=\frac{1}{T} \sum_{t=1}^{T} E_{t-1} G_{t}^{H}+\frac{1}{T} \sum_{t=1}^{T}\left(G_{t}^{H}-E_{t-1} G_{t}^{H}\right) .
$$

By virtue of (21) and (26), for each $H$ the sequence of random variables $G_{t}^{H}\left(s^{t}\right)$ is bounded uniformly in $t$ and $\left(s_{t}\right)_{t \geq 1}$. Consequently, we can apply the strong law of large numbers for martingale differences (see, e.g., [15]), which yields

$$
\frac{1}{T} \sum_{t=1}^{T}\left(G_{t}^{H}-E_{t-1} G_{t}^{H}\right) \rightarrow 0 \text { (a.s.). }
$$


Therefore,

$$
\liminf _{t \rightarrow \infty} T^{-1} \sum_{t=1}^{T} G_{t}^{H} \geq \delta
$$

which proves (25).

It remains to prove the uniqueness of a symmetric a.s. equilibrium. Suppose a strategy $\lambda \neq \lambda^{*}$ forms such an equilibrium. Then

$$
\left.0=\xi^{i}\left(s^{\infty} ; \lambda, \ldots, \lambda\right) \geq \xi^{i}\left(s^{\infty} ; \lambda, \ldots, \lambda, \lambda^{*}\right) \text { (a.s. }\right),
$$

where

$$
\xi^{i}\left(s^{\infty} ; \lambda, \ldots, \lambda, \lambda^{*}\right)=\limsup _{t \rightarrow \infty} t^{-1} \ln \frac{r_{t}^{N}}{1-r_{t}^{N}} .
$$

As we have shown above that

$$
\liminf _{t \rightarrow \infty} t^{-1} \ln \frac{r_{t}^{N}}{1-r_{t}^{N}}>0 \text { (a.s.), }
$$

which yields the inequality " $<"$ in $(28)$. This is a contradiction.

\section{Conclusion}

In conventional stochastic dynamic games and Dynamic Stochastic General Equilibrium, models are based on the classical utility maximization paradigm. This paradigm is central to both fundamental concepts: Nash equilibrium and Walrasian equilibrium. To make justified quantitative recommendations using such models one needs to have information about the individual utilities of market players, which are typically unobservable. Furthermore, in a stochastic context computations must rely upon the knowledge of unobservable individual beliefs (subjective probabilities). Evolutionary Finance (EF) abandons these restrictive hypotheses, which makes the theory closer to practical applications.

The present study makes an attempt to establish links between the EF models and the classical ones. We have found a way of formulating an evolutionary solution concept - a strategy strictly dominating the market at an exponential rate - in terms of a non-conventional version of Nash equilibrium holding a.s. It turns out that this can be done by introducing as the objective function the Lyapunov exponent of the relative growth of wealth of a market participant. Here, "relative" refers to the ratio of wealth of the market player and the total wealth of the group of her rivals. By replacing the standard Nash equilibrium conditions formulated in terms of expected utilities by their versions holding almost surely, we obtain a solution concept that happens to be equivalent to the exponential domination of the market.

The analogous results for the EF model with long-lived assets were obtained in [3]. Here, the goal is achieved for another core EF model, the one with shortlived assets. Thus, this study fills a gap in the theory that existed for about a decade. 
An interesting topic for further research would be the question of the existence of asymmetric Nash equilibria. To approach this question one has to develop techniques for the analysis of the dynamical system (23) when none of the strategies coincides with $\lambda^{*}$. Up to now no substantial progress on this problem (which is of interest as such) has been achieved.

\section{Appendix}

Proof of Lemma 1. Let us first assume that at least one of the coordinates of $\lambda$ is zero, i.e. $\mathbf{K}:=\left\{k: \lambda_{k}=0\right\} \neq \emptyset$. Denote by $S^{\prime}$ the set of those $s$ for which $\sum_{k=1}^{K} R_{k}(s) \lambda_{k}=0$. If $s \in S^{\prime}$, then $R_{k}(s) \lambda_{k}=0$ for all $k$. Consequently,

$$
B=\sum_{k=1}^{K} R_{k}(s) \frac{\lambda_{k}}{\lambda_{k}^{*} \kappa+\lambda_{k}(1-\kappa)}=0, s \in S^{\prime},
$$

and so $F(\lambda, \kappa ; s)=+\infty, s \in S^{\prime}$. Thus for any $H>1$, we have

$$
\begin{gathered}
E \min [H, \ln F(\lambda, \kappa ; s)] \\
=E \min [H, \ln F(\lambda, \kappa ; s)] \mathbf{1}_{S^{\prime}}+E \min [H, \ln F(\lambda, \kappa ; s)] \mathbf{1}_{S \backslash S^{\prime}} \geq \\
H P\left(S^{\prime}\right)+2(\ln c)\left(1-P\left(S^{\prime}\right)\right),
\end{gathered}
$$

where $\mathbf{1}_{\Gamma}$ is the indicator function of the set $\Gamma$.

Assume that $P\left(S^{\prime}\right)>0$. Then for all $H$ large enough, the expression in (29) is greater than 1 . The assertion of Lemma 1 will be true for any $H$ for which (29) is greater than 1 and $\delta=1$.

Now let us assume that $P\left(S^{\prime}\right)=0$, i.e. $\sum_{k=1}^{K} R_{k}(s) \lambda_{k}>0$ (a.s.). In this case, we will conduct the proof of the lemma in three steps.

Step 1: Let us prove that

$$
E \ln F(\lambda, \kappa ; s)>0 \text { for all } \kappa \in(0,1] .
$$

By applying Jensen's inequality, we find

$$
\begin{gathered}
E \ln \sum_{k=1}^{K} R_{k}(s) \frac{\lambda_{k}^{*}}{\lambda_{k}^{*} \kappa+\lambda_{k}(1-\kappa)} \geq E \sum_{k=1}^{K} R_{k}(s) \ln \frac{\lambda_{k}^{*}}{\lambda_{k}^{*} \kappa+\lambda_{k}(1-\kappa)} \\
=\sum_{k=1}^{K} \lambda_{k}^{*} \ln \frac{\lambda_{k}^{*}}{\lambda_{k}^{*} \kappa+\lambda_{k}(1-\kappa)}
\end{gathered}
$$

and

$$
\begin{gathered}
E \ln \sum_{k=1}^{K} R_{k}(s) \frac{\lambda_{k}}{\lambda_{k}^{*} \kappa+\lambda_{k}(1-\kappa)}<\ln E \sum_{k=1}^{K} R_{k}(s) \frac{\lambda_{k}}{\lambda_{k}^{*} \kappa+\lambda_{k}(1-\kappa)} \\
=\ln \sum_{k=1}^{K} \lambda_{k}^{*} \frac{\lambda_{k}}{\lambda_{k}^{*} \kappa+\lambda_{k}(1-\kappa)} .
\end{gathered}
$$


The inequality in (33) is strict because the right-hand side of (33) is finite (all $\lambda_{k}^{*}$ and some $\lambda_{k}$ are strictly positive) and there is no constant $\gamma$ such that

$$
\sum_{k=1}^{K} R_{k}(s) \frac{\lambda_{k}}{\lambda_{k}^{*} \kappa+\lambda_{k}(1-\kappa)}=\gamma(\text { a.s. }) \text {. }
$$

Indeed, if (35) holds, then

$$
\sum_{k=1}^{K} R_{k}(s) \nu_{k}=0 \text { (a.s.) }
$$

where $\nu_{k}:=\lambda_{k}\left[\lambda_{k}^{*} \kappa+\lambda_{k}(1-\kappa)\right]^{-1}-\gamma$. Observe that at least one of the numbers $\nu_{k}$ is not equal to zero. Otherwise, $\lambda_{k}=\left[\lambda_{k}^{*} \kappa+\lambda_{k}(1-\kappa)\right] \gamma$ for all $k$, and by summing up these equalities over $k$, we get $\gamma=1$, which yields $\lambda_{k}=\left[\lambda_{k}^{*} \kappa+\lambda_{k}(1-\kappa)\right]$. Thus $\lambda_{k}=\lambda_{k}^{*}$ for all $k$ (recall that $\kappa \neq 0$ ). This is a contradiction because $\lambda \neq \lambda^{*}$. From this we conclude that at least one of the numbers $v_{k}$ is not equal to zero, which shows that (36) cannot hold since the functions $R_{k}(s)$ are linearly independent with respect to the given distribution on $S$.

By combining (31)-(34), we get

$$
E \ln F(\lambda, \kappa ; s)>\Phi_{\kappa}(\lambda),
$$

where

$$
\Phi_{\kappa}(\lambda):=\sum_{k=1}^{K} \lambda_{k}^{*} \ln \frac{\lambda_{k}^{*}}{\lambda_{k}^{*} \kappa+\lambda_{k}(1-\kappa)}-\ln \sum_{k=1}^{K} \lambda_{k}^{*} \frac{\lambda_{k}}{\lambda_{k}^{*} \kappa+\lambda_{k}(1-\kappa)} .
$$

In [9], pp. 337-338, it was proved that $\Phi_{\kappa}(\lambda)>0$ for all $\lambda=\left(\lambda_{1}, \ldots, \lambda_{K}\right)>0$. Therefore $\Phi_{\kappa}\left(\lambda(1-\varepsilon)+\varepsilon \lambda^{*}\right)>0$ for each $\varepsilon>0$. The function $\Phi_{\kappa}(\lambda)$ is finite and continuous on $\Delta^{K}$. Consequently, $\left.\Phi_{\kappa}(\lambda)=\lim _{\varepsilon \downarrow 0} \Phi_{\kappa}\left(\lambda(1-\varepsilon)+\varepsilon \lambda^{*}\right)\right) \geq 0$. This inequality combined with $(37)$ yields $(30)$.

Step 2: Let us show that for all $\kappa \in(0,1]$,

$$
F(\lambda, \kappa, s) \geq F(\lambda, 1, s)=\left[\sum_{k=1}^{K} R_{k}(s) \frac{\lambda_{k}}{\lambda_{k}^{*}}\right]^{-1}
$$

$\left(0^{-1}:=+\infty\right)$. Fix any $s \in S$. If $R_{k}(s) \lambda_{k}=0$ for all $k$, then $F(\lambda, \kappa, s)=$ $F(\lambda, 1, s)=+\infty$, and so inequality (38) holds. Let us assume that $R_{k}(s) \lambda_{k} \neq 0$ for some $k$. Then the denominator in (20) is strictly positive, and so $c^{2} \leq$ $F(\lambda, \kappa, s)<\infty$. For $\kappa \in(0,1)$, we have $F(\lambda, \kappa ; s)=G(\lambda, u, s)$, where

$$
G(\lambda, u, s):=\frac{\sum_{k=1}^{K} R_{k}(s) \frac{\lambda_{k}^{*}}{\lambda_{k}^{*} u+\lambda_{k}}}{\sum_{k=1}^{K} R_{k}(s) \frac{\lambda_{k}}{\lambda_{k}^{*} u+\lambda_{k}}}=\frac{\sum_{k=1}^{K} R_{k}(s) \frac{\lambda_{k}^{*}}{\lambda_{k}^{*}+\lambda_{k} / u}}{\sum_{k=1}^{K} R_{k}(s) \frac{\lambda_{k}}{\lambda_{k}^{*}+\lambda_{k} / u}},
$$


and $u:=\kappa(1-\kappa)^{-1}$. When the variable $\kappa$ ranges through $(0,1)$, the variable $u$ ranges through $(0,+\infty)$, and $G(\lambda, u, s) \rightarrow\left[\sum_{k=1}^{K} R_{k}(s)\left(\lambda_{k} / \lambda_{k}^{*}\right)^{-1}\right]^{-1}$ as $u \rightarrow$ $\infty$. Thus, to verify (38), it is sufficient to show that the derivative $G(\lambda, u ; s)^{\prime}$ of the function $G(\lambda, u ; s)$ with respect to $u$ is non-positive for all $u>0$. Assuming that the parameter $s$ is fixed, we omit " $s$ " in the notation and write

$$
\begin{gathered}
G(\lambda, u)^{\prime}= \\
=\frac{\left(\sum R_{k} \frac{\lambda_{k}^{*}}{\lambda_{k}^{*} u+\lambda_{k}}\right)^{\prime}\left(\sum R_{k} \frac{\lambda_{k}}{\lambda_{k}^{*} u+\lambda_{k}}\right)-\left(\sum R_{k} \frac{\lambda_{k}^{*}}{\lambda_{k}^{*} u+\lambda_{k}}\right)\left(\sum R_{k} \frac{\lambda_{k}}{\lambda_{k}^{*} u+\lambda_{k}}\right)^{\prime}}{\left(\sum R_{k} \frac{\lambda_{k}}{\lambda_{k}^{*} u+\lambda_{k}}\right)^{2}},
\end{gathered}
$$

where $\sum=\sum_{k=1}^{K}$. The sign of the above fraction is the same as the sign of its nominator

$$
\begin{aligned}
J & :=-\sum R_{k} \frac{\left(\lambda_{k}^{*}\right)^{2}}{\left(\lambda_{k}^{*} u+\lambda_{k}\right)^{2}} \sum R_{k} \frac{\lambda_{k}}{\lambda_{k}^{*} u+\lambda_{k}} \\
& +\sum R_{k} \frac{\lambda_{k}^{*}}{\lambda_{k}^{*} u+\lambda_{k}} \sum R_{k} \frac{\lambda_{k} \lambda_{k}^{*}}{\left(\lambda_{k}^{*} u+\lambda_{k}\right)^{2}}
\end{aligned}
$$

By setting $w_{k}:=\lambda_{k}^{*} u+\lambda_{k}$ and $v_{k}:=\lambda_{k}^{*} w_{k}^{-1}$, we obtain $\lambda_{k}=w_{k}-\lambda_{k}^{*} u$ and

$$
\begin{gathered}
J=-\sum R_{k}\left(\lambda_{k}^{*}\right)^{2} w_{k}^{-2} \sum R_{k} \lambda_{k} w_{k}^{-1}+\sum R_{k} \lambda_{k}^{*} w_{k}^{-1} \sum R_{k} \lambda_{k} \lambda_{k}^{*} w_{k}^{-2} \\
=-\sum R_{k}\left(\lambda_{k}^{*}\right)^{2} w_{k}^{-2} \sum R_{k}\left(w_{k}-\lambda_{k}^{*} u\right) w_{k}^{-1} \\
+\sum R_{k} \lambda_{k}^{*} w_{k}^{-1} \sum R_{k}\left(w_{k}-\lambda_{k}^{*} u\right) \lambda_{k}^{*} w_{k}^{-2} \\
=-\sum R_{k} v_{k}^{2} \sum\left(R_{k}-R_{k} u v_{k}\right)+\sum R_{k} v_{k} \sum R_{k}\left(v_{k}-v_{k}^{2} u\right) \\
-\sum R_{k} v_{k}^{2}\left(1-\sum R_{k} u v_{k}\right)+\sum R_{k} v_{k}\left(\sum R_{k} v_{k}-\sum R_{k} v_{k}^{2} u\right) \\
=-\sum R_{k} v_{k}^{2}+\left(\sum R_{k} v_{k}^{2}\right) \sum R_{k} v_{k} u+\left(\sum R_{k} v_{k}\right)^{2}-\left(\sum R_{k} v_{k}\right) \sum R_{k} v_{k}^{2} u \\
=\left(\sum R_{k} v_{k}\right)^{2}-\sum R_{k} v_{k}^{2} \leq 0 .
\end{gathered}
$$

The last expression is non-positive by virtue of the Schwartz inequality (we use here the fact that $R_{k} \geq 0$ and $\sum R_{k}=1$ ). This completes the proof of inequality (38).

Step 3. Let us show that there exists a natural number $m$ such that $E \min \{m, \ln F(\lambda, 1, s)\}>0$. The sequence of random variables

$$
\phi_{m}:=\min \{m, \ln F(\lambda, 1, s)\}, m=1,2, \ldots,
$$

is bounded below by $2 \ln c$ (see (21)), is nondecreasing and tends to $\ln F(\lambda, 1, s)$ for each $s$. Consequently, we have $E \phi_{m} \rightarrow E \ln F(\lambda, 1, s)>0$ (see (30)), and so 
$E \phi_{m}>0$ for some $m=m_{0}$. By setting $H:=m_{0}, \delta:=E \min \{H, \ln F(\lambda, 1 ; s)\}>$ 0 and using (38), we find

$$
E \min \{H, \ln F(\lambda, \kappa ; s)\} \geq E \min \{H, \ln F(\lambda, 1 ; s)\}=\delta,
$$

which proves Lemma 1 in the case when at least one of the coordinates of $\lambda$ is zero.

Now assume that $\lambda$ has no zero coordinates: $\lambda_{k}>0$ for each $k$. Then the function $\ln F(\lambda, \kappa ; s), \kappa \in[0,1]$, is uniformly bounded:

$$
2 \ln c \leq \ln F(\lambda, \kappa ; s) \leq \ln \left(\min _{k} \lambda_{k}\right)^{-2}
$$

and so $E \ln F(\lambda, \kappa ; s)$ is continuous in $\kappa \in[0,1]$. It suffices to show that the infimum of $E \ln F(\lambda, \kappa ; s)$ with $\kappa \in[0,1]$ is strictly positive (then $\delta$ can be defined as this infimum and $H$ as $\left.2|\ln c|+2\left|\ln \min \lambda_{k}\right|\right)$. In view of the continuity of $E \ln F(\lambda, \kappa ; s)$ this will be proved if we establish the inequality $E \ln F(\lambda, \kappa ; s)>0$ for each $\kappa \in[0,1]$. For $\kappa \in(0,1]$ this was proved above (see (30)) under the condition that $\sum_{k=1}^{K} R_{k}(s) \lambda_{k}>0$ (a.s.), which holds if $\lambda>0$. In the case of $\kappa=0$, we have

$$
E \ln F(\lambda, 0, s)=E \ln \sum_{k=1}^{K} R_{k}(s) \frac{\lambda_{k}^{*}}{\lambda_{k}} \geq \ln \sum_{k=1}^{K} \lambda_{k}^{*} \frac{\lambda_{k}^{*}}{\lambda_{k}} \geq \sum_{k=1}^{K} \lambda_{k}^{*} \ln \frac{\lambda_{k}^{*}}{\lambda_{k}}>0
$$

as long as $\lambda^{*}, \lambda>0$ and $\lambda \neq \lambda^{*}$.

\section{References}

1. Algoet, P.H., and Cover, T.M. (1988). Asymptotic optimality and asymptotic equipartition properties of log-optimum investment. Annals of Probability 16(2), 876-898.

2. Amir, R., I.V. Evstigneev, and Schenk-Hoppé, K.R. (2013). Asset Market Games of Survival: A Synthesis of Evolutionary and Dynamic Games. Annals of Finance 9(2), 121-144.

3. Bahsoun, W., Evstigneev, I.V., and Xu, L. (2011). Almost Sure Nash Equilibrium Strategies in Evolutionary Models of Asset Markets. Mathematical Methods of Operations Research 73, 235-250.

4. Bell, R.M., and Cover, T.M. (1980). Competitive optimality of logarithmic investment. Mathematics of Operations Research 5(2), 161-166.

5. Bell, R.M., and Cover, T.M. (1988). Game-theoretic optimal portfolios. Management Science 34(6), 724-733.

6. Breiman, L. (1961). Optimal gambling systems for favorable games. Proceedings of the Fourth Berkeley Symposium on Mathematical Statistics and Probability 1, 65-78.

7. Cover, T.M. (1998). Shannon and investment. IEEE Information Theory Society Newsletter, Summer, Special Golden Jubilee Issue, 10-11.

8. Cover, T.M., and Thomas, J.A. (2006). Elements of Information Theory, 2nd Edition, Wiley.

9. Evstigneev, I.V., Hens, T., and Schenk-Hoppé, K.R. (2002). Market selection of financial trading strategies: Global stability. Mathematical Finance 12(4), 329-339.

10. Evstigneev, I.V., Hens, T., and Schenk-Hoppé, K.R. (2006). Evolutionary stable stock markets. Economic Theory 27(2), 449-468.

11. Evstigneev, I.V., Hens, T., and Schenk-Hoppé, K.R. (2009). Evolutionary finance. Handbook of Financial Markets: Dynamics and Evolution (T. Hens and K.R. Schenk-Hoppé, eds.), Chapter 9, Amsterdam: Elsevier, 507-566. 
12. Evstigneev, I.V., Hens, T., and Schenk-Hoppé, K.R. (2015). Mathematical Financial Economics: A Basic Introduction. Springer.

13. Evstigneev, I.V., Hens, T., and Schenk-Hoppé, K.R. (2016). Evolutionary behavioural finance. Handbook of Post Crisis Financial Modelling (E. Haven et al., eds.), Palgrave MacMillan, 214-234.

14. Hakansson, N.H., and Ziemba, W.T. (1995). Capital growth theory. Handbooks in Operations Research and Management Science (R.A. Jarrow et al., eds.), Amsterdam: Elsevier, 65-86.

15. Hall, P., and Heyde, C.C. (1980). Martingale limit theory and its application. London: Academic Press.

16. Kelly, J.L. (1956). A new interpretation of information rate. Bell System Technical Journal 35(7), 917-926.

17. Kuhn, D., and Luenberger, D.G. (2010). Analysis of the rebalancing frequency in logoptimal portfolio selection. Quantitative Finance 10, 221-234.

18. Latané, H. (1959). Criteria for choice among risky ventures. Journal of Political Economy $67,144-155$

19. MacLean, L.C., Thorp, E.O., and Ziemba, W.T., eds. (2010). The Kelly Capital Growth Investment Criterion: Theory and Practice. Singapore: World Scientific.

20. MacLean, L.C., and Ziemba, W.T., eds. (2013). Handbook of the Fundamentals of Financial Decision Making. Singapore: World Scientific.

21. Ziemba, W.T. and Vickson, R.G. (2011). Models of optimal capital accumulation and portfolio selection and the capital growth criterion. The Kelly Capital Growth Investment Criterion: Theory and Practice (L.C. MacLean et al., eds), Singapore: World Scientific, 473-485. 